\title{
Disorder, cluster spin glass, and hourglass spectra in striped magnetic insulators
}

\author{
Eric C. Andrade and Matthias Vojta \\ Institut für Theoretische Physik, Technische Universität Dresden, 01062 Dresden, Germany
}

(Dated: November 9, 2018)

\begin{abstract}
Hourglass-shaped magnetic excitation spectra have been detected in a variety of doped transitionmetal oxides with stripe-like charge order. Compared to the predictions of spin-wave theory for perfect stripes, these spectra display a different intensity distribution and anomalous broadening. Here we show, based on a comprehensive modelling for $\mathrm{La}_{5 / 3} \mathrm{Sr}_{1 / 3} \mathrm{CoO}_{4}$, how quenched disorder in the charge sector causes frustration, and consequently cluster-glass behavior at low temperatures, in the spin sector. This spin-glass physics, which is insensitive to the detailed nature of the charge disorder, but sensitive to the relative strength of the magnetic inter-stripe coupling, ultimately determines the distribution of magnetic spectral weight: The excitation spectrum, calculated using spin waves in finite disordered systems, is found to match in detail the observed hourglass spectrum.
\end{abstract}

Magnetic excitations, as measured by inelastic neutron scattering (INS), serve as a fingerprint of the underlying magnetic state. In layered transition-metal oxides, socalled "hourglass" spectra are frequently observed [1-10], consisting of intensity at incommensurate (IC) wavevectors at low energy which, with increasing energy, first disperses towards the antiferromagnetic $(\mathrm{AF})$ wavevector $(\pi, \pi)$, leading to a large-intensity "resonance", and then disperses outwards again. In the case of cuprate superconductors, explanations both in terms of weak-coupling excitations of a Fermi liquid and of strong-coupling collective modes of an underlying stripe state have been proposed [11-15].

More recently, hourglass spectra have been detected in doped cobaltates and manganites [9, 10]. Because these are insulators, the magnetic excitations can be conclusively linked to collective local-moment physics. Moreover, charge order is known to occur, i.e., magnetic and non-magnetic ions form a pattern of unidirectional twodimensional (2D) stripes [16]. Indeed, assuming magnetic moments to be arranged in stripes and coupled by superexchange interactions allows one to qualitatively understand some gross features of the measured spectra.

However, the experimental data display pronounced deviations from the spin-wave spectra of perfect stripes: (i) At low energies, outward dispersing excitation branches are essentially completely missing. (ii) The data typically show a large and anisotropic intrinsic broadening. (iii) For a sizeable range of intermediate energies a "vertical dispersion" is observed near $(\pi, \pi)$. (iv) Compared to perfect-stripe spin-wave results, spectral weight is redistributed from higher energies into the broad "resonance" (or saddle-point) feature. It has been suggested that quenched disorder plays a role in explaining these features, but concrete calculations have been restricted to ad-hoc broadening of perfect-stripe results, and deeper insights are lacking $[9,10,16]$.

In this paper we analyse spin-wave spectra of stripes in $2 \mathrm{D}$ insulators, treating disorder exactly in large finite-size systems. Importantly, stripe disorder generically leads to magnetic frustration, because charge stripes act as antiphase domain walls for the AF order. Our results identify the hourglass as the excitation spectrum of the low-temperature cluster-spin-glass state which emerges due to the disorder-induced frustration. We discuss the ingredients required for this spin-glass physics and the implications for different material families.

$\mathrm{La}_{5 / 3} \mathrm{Sr}_{1 / 3} \mathrm{CoO}_{4}$. For definiteness, we choose to model the magnetism in $\mathrm{La}_{5 / 3} \mathrm{Sr}_{1 / 3} \mathrm{CoO}_{4}$, for which comprehensive INS data is available [9]. $\mathrm{La}_{2-x} \mathrm{Sr}_{x} \mathrm{CoO}_{4}$ shows signatures of stripe order for $0.3<x<0.6[16,17]$. For $x=1 / 3$ the $\mathrm{Co}^{2+}$ and $\mathrm{Co}^{3+}$ ions form a robust diagonal period-3 stripe pattern, with charge order setting in at $T_{\mathrm{CO}}$ far above room temperature $[17,18] . \mathrm{Co}^{2+}$ is in a $S=3 / 2$ high-spin state, while $\mathrm{Co}^{3+}$ is in a $S=0$ lowspin state $[9,19]$, and from the properties of the $x=0$ [20] and $x=0.5$ [21] compounds it is known that the magnetic couplings $-J$ between neighboring $\mathrm{Co}^{2+}$ and $J^{\prime}$ across a non-magnetic $\mathrm{Co}^{3+}-$ are both $\mathrm{AF}$. For perfect charge order, this results in a stripe pattern as depicted in Fig. 1(a), exhibiting IC magnetism with magnetic Bragg peaks at $\vec{Q}_{\mathrm{S}}=(\pi \pm \pi / 3, \pi \mp \pi / 3)$. [Stripes running along the opposite diagonal have $\vec{Q}_{\mathrm{S}}=(\pi \pm \pi / 3, \pi \pm \pi / 3)$.]

Disordered charge stripes. The experiments in Ref. 9 have proven substantial disorder effects to be present in $\mathrm{La}_{5 / 3} \mathrm{Sr}_{1 / 3} \mathrm{CoO}_{4}$. It is plausible to assume that those originate from static imperfections in the charge order. In contrast, dynamic fluctuations of the charge order are likely negligible for $T \ll T_{\mathrm{CO}}$ because of the robust insulating nature of the material which is re-enforced by spin-blockade effects [16, 17, 22, 23].

We therefore start by modelling stripes of $\mathrm{Co}^{2+}$ and $\mathrm{Co}^{3+}$ ions. We construct an Ising model for variables $n_{i}$ on the sites $i$ of a square lattice, where $n_{i}=0$ refers to a $\mathrm{Co}^{2+}$ and $n_{i}=1$ to a $\mathrm{Co}^{3+}$ ion. The model is chosen such that it has perfect stripe configurations with ordering wavevector $\vec{Q}_{\mathrm{C}}=(\pi \pm \pi / 3, \pi \mp \pi / 3)$ [as in Fig. $1(\mathrm{a})]$ and $\vec{Q}_{\mathrm{C}}=(\pi \pm \pi / 3, \pi \pm \pi / 3)$ as ground states at fixed filling $\langle n\rangle=1 / 3$, for details see Ref. 24 .

Monte-Carlo (MC) simulations of this model on $L \times L$ lattices $(L \leq 48)$ are used to generate charge configurations away from this ordered state, i.e., with well-defined 
(a)

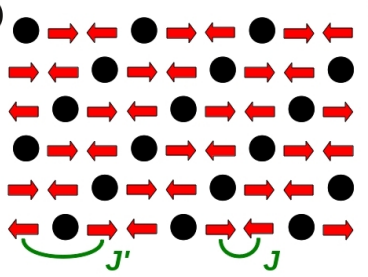

(c)

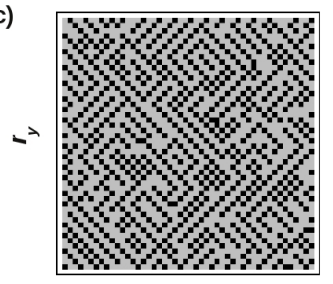

$r_{x}$

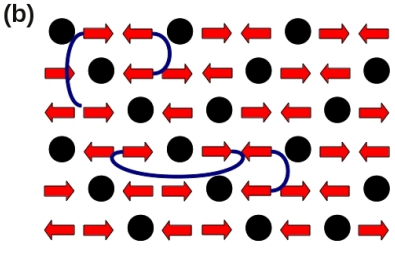

(d)

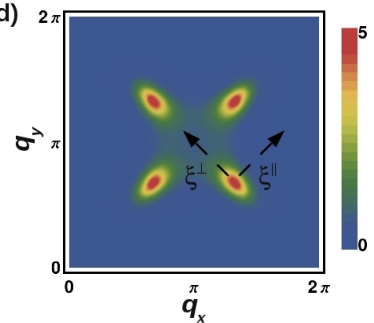

Figure 1: (a) Perfect stripe order in the $\mathrm{CoO}_{2}$ planes of $\mathrm{La}_{5 / 3} \mathrm{Sr}_{1 / 3} \mathrm{CoO}_{4}$. Circles (arrows) represent non-magnetic $\mathrm{Co}^{3+}$ (magnetic $\mathrm{Co}^{2+}$ ) ions. Both magnetic couplings $J$ and $J^{\prime}$ are AF. (b) Frustrated bonds caused by disorder in the charge sector. (c) Disordered charge configuration with $\xi_{\mathrm{C}}^{\|} \approx 5$ and $\xi_{\mathrm{C}}^{\perp} \approx 2$, obtained from the Ising model [24] for $L=48$. Black (gray) squares correspond to $\mathrm{Co}^{3+}\left(\mathrm{Co}^{2+}\right)$ ions. (d) Charge structure factor $S_{\mathrm{C}}(\vec{q})$ for the same parameters as in (c) averaged over $10^{3}$ charge configurations.

short-range order [24]. Representative results are in Fig. 1 (c) and Ref. 24, showing multiple stripe domains and various types of defects. We characterize these configurations by their correlation lengths $\xi_{\mathrm{C}}^{\|}\left(\xi_{\mathrm{C}}^{\perp}\right)$ parallel (perpendicular) to the stripe direction, we obtain the $\xi_{\mathrm{C}}$ from the charge structure factor $S_{\mathrm{C}}(\vec{q})$, Fig. $1(\mathrm{~d})$, by a fit to a Lorentzian lineshape near $\vec{Q}_{\mathrm{C}}$. We note that the procedure of generating disordered stripes is not unique, as - for fixed $\xi_{\mathrm{C}}$ - the types and distributions of defects will depend on details of both the Ising model and the MC protocol [24]. However, such details have remarkably little influence on the behavior of the spin sector.

Spin model. To model the magnetism of $\mathrm{La}_{5 / 3} \mathrm{Sr}_{1 / 3} \mathrm{CoO}_{4}$, we place localized $S=1 / 2$ spins on the $\mathrm{Co}^{2+}$ sites of a $2 \mathrm{D}$ disordered stripe configuration as in Fig. 1(b,c). The spins are assumed to interact via Heisenberg exchanges as in Fig. 1(a):

$$
\mathcal{H}_{\mathrm{sp}}=\sum_{i, j} \sum_{\alpha} J_{i j}^{\alpha} S_{i}^{\alpha} S_{j}^{\alpha}
$$

The first sum runs over the lattice sites with $n_{i}=0$ according to a given charge configuration $\left\{n_{i}\right\}$, and $\alpha=$ $x, y, z$. Guided by Refs. 9, 20, 21 we assume that the nearest-neighbor coupling $J$ (which sets our energy scale) has the same form as in the undoped parent compound: $J^{x}=J(1+\epsilon), J^{y}=J$, and $J^{z}=J(1-\delta)$. The parameters $\delta$ and $\epsilon$ control the spin anisotropy with $\delta=0.28$ and $\epsilon=0.013[20,21,24]$. Because the coupling across a $\mathrm{Co}^{3+}$ ion, $J^{\prime}$, is considerably smaller than $J$, we assume

it to be isotropic with typically $J^{\prime}=0.05 J$. Interlayer couplings are neglected.

Spin-glass ground state and excitations. For imperfect charge order, $\mathcal{H}_{\mathrm{sp}}$ describes frustrated magnetism: As illustrated in Fig. 1(b), stripe defects generically lead to frustrated couplings between neighboring AF domains. This combination of disorder and frustration is expected to lead to spin-glass behavior, more precisely cluster spinglass behavior, because the entities subject to frustrated couplings are not single spins, but domains of varying size. This spin glass displays short-range magnetic order (inherited from the perfect stripe state), with magnetic correlation lengths $\xi_{\mathrm{S}}^{\|}, \xi_{\mathrm{S}}^{\perp}$ which we determine from the spin structure factor $S_{\mathrm{S}}(\vec{q})$. In general, we find that $\xi_{\mathrm{S}}$ follows $\xi_{\mathrm{C}}$ [24], but for a strongly disordered charge sector with $\xi_{\mathrm{C}}^{\|} \lesssim 4, S_{\mathrm{S}}(\vec{q})$ is no longer peaked at the IC $\vec{Q}_{\mathrm{S}}$, but instead displays a broad maximum at $(\pi, \pi)$. This implies that the real-space period-3 structure is lost, and a spin glass with very short-ranged AF correlations emerges.

To access the spin dynamics, we first determine locally stable classical states of $\mathcal{H}_{\mathrm{sp}}$ for a given charge configuration $\left\{n_{i}\right\}$ : For classical spins $\vec{S}_{i}$, we perform MC simulations of $\mathcal{H}_{\mathrm{sp}}$ utilizing single-site updates, combining the heat-bath and microcanonical (or over-relaxation) methods. To efficiently sample all spin configurations, we also employ the parallel-tempering algorithm [25]. This procedure is followed by a quench down to $T=0$ using a greedy conjugate-gradient algorithm. Our simulations confirm spin-glass behavior, as detailed in Ref. 24 .

We then calculate the excitation spectrum of $\mathcal{H}_{\text {sp }}$ using linear spin-wave theory on finite lattices: Deviations from a classical state are represented by non-interacting bosons [24] modelling Gaussian magnetic fluctuations. The dynamic susceptibility is given by:

$$
\chi^{\prime \prime}(\vec{q}, \omega)=\left[\sum_{\alpha} \sum_{\nu}\left|\left\langle 0\left|S^{\alpha}(\vec{q})\right| \nu\right\rangle\right|^{2} \delta\left(\omega-\omega_{\nu}\right)\right]_{a v},
$$

where $S^{\alpha}(\vec{q})$ is the Fourier-transformed spin operator, $|0\rangle$ the magnon vacuum, and $|\nu\rangle$ a single-magnon state with energy $\omega_{\nu}$. We typically evaluate the average $[\cdots]_{a v}$ over 80 spin states obtained from 40 charge configurations. Formally, such a calculation yields the zero-temperature excitation spectrum of a system with spatially disordered static stripes, but the results continue to apply at finite temperatures $T$ and even for slowly fluctuating stripes, provided that both temperature and fluctuation frequency are small compared to the energies $\omega$ of interest in $\chi^{\prime \prime}(\vec{q}, \omega)$.

Charge disorder and hourglass spectrum. Fig. 2 shows our central result, namely the evolution of $\chi^{\prime \prime}(\vec{q}, \omega)$ with varying degree of stripe disorder for weak inter-stripe coupling (i.e. large $J / J^{\prime}$ ). The perfect-stripe case Fig. 2(a) has sharp magnon peaks; at low energies gapped magnon branches (recall the magnetic anisotropy) disperse both inward and outward from $\vec{Q}_{\mathrm{S}}$, and a sharply 

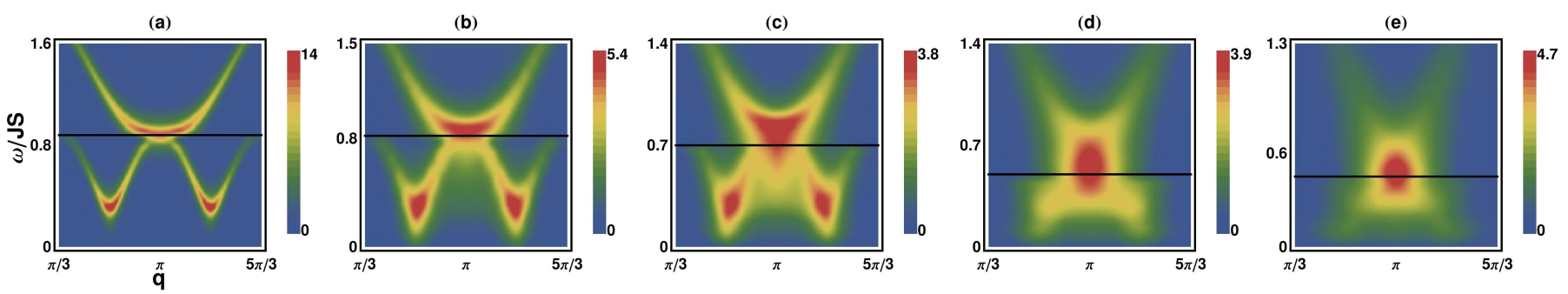

Figure 2: Magnetic excitations, $\chi^{\prime \prime}(\vec{q}, \omega)$, of disordered stripes. From (a) to (e) the disorder in the charge sector increases, as characterized by the correlation lengths $\xi_{\mathrm{C}}^{\|}, \xi_{\mathrm{C}}^{\perp}$. Each panel is divided at $\omega=\omega_{\mathrm{S}}$, with the lower [upper] portion showing data along $\vec{q}=(q, q)[(q, \pi)]$ in order to represent the high-intensity features. Parameters are $J^{\prime}=0.05 J, L=36$, and (a) Perfect stripes, $\omega_{\mathrm{S}}=0.88 J S ;(\mathrm{b}) \xi_{\mathrm{C}}^{\|} \simeq 19, \xi_{\mathrm{C}}^{\perp} \simeq 8, \omega_{\mathrm{S}}=0.82 J S ;(\mathrm{c}) \xi_{\mathrm{C}}^{\|} \simeq 10, \xi_{\mathrm{C}}^{\perp} \simeq 4, \omega_{\mathrm{S}}=0.70 J S ;(\mathrm{d}) \xi_{\mathrm{C}}^{\|} \simeq 5, \xi_{\mathrm{C}}^{\perp} \simeq 2, \omega_{\mathrm{S}}=0.50 J S$; (e) $\xi_{\mathrm{C}}^{\|} \simeq 3, \xi_{\mathrm{C}}^{\perp} \lesssim 1, \omega_{\mathrm{S}}=0.45 J S[24]$. The $\delta$ peaks in Eq. (2) were replaced by Lorentzians with width $\Gamma=0.1 J S$.

peaked large intensity maximum occurs at $(\pi, \pi)$ corresponding to a saddle point of the spin-wave dispersion. In contrast, as disorder increases, Figs. 2(b,c), the excitations are broadened both in momentum and energy. The anisotropy gap is smeared, and the intensity at low energies is enhanced as compared to the clean case: Disorder causes a strong redistribution of spectral weight from high to low energies [24]. Even more strikingly, for the strongly disordered case in Figs. 2(d,e), the outward dispersing branches at low energy have disappeared completely. Instead, the weight is concentrated near $(\pi, \pi)$ over a broad energy range, leading to an apparent "vertical" dispersion. Finally, in Fig. 2(e) the charge disorder is so strong that the IC signal at small $\omega$ is hardly visible; here, $S_{\mathrm{S}}(\vec{q})$ shows a broad maximum at $(\pi, \pi)$ [24].

A remarkable trend is that disorder causes - apart from broadening - a strong downward renormalization of the saddle-point energy: For otherwise identical parameters, $\omega_{\mathrm{S}}$ in Fig. 2(e) is half of that in the perfect-stripe case.

Influence of model parameters. In the spin sector, the only parameters are the exchange constants $J$ and $J^{\prime}$ and their anisotropies, whose values we fixed based on experimental data on $\mathrm{La}_{5 / 3} \mathrm{Sr}_{1 / 3} \mathrm{CoO}_{4}$ [9] and its parent compound [20]. Some uncertainty concerns the strength of the inter-stripe coupling, i.e., the ratio $J / J^{\prime}$. In fact, this ratio varies considerably between different families of striped oxides, with values of 2 reported for nickelates (which do not show an hourglass dispersion) [26, 27] and 7-20 for cobaltates [9]. Spin-wave calculations in the disorder-free case $[9,26,28]$ show that large $J / J^{\prime}$ is crucial to the observation of an hourglass-like spectrum, whereas for small $J / J^{\prime}$ the saddle point moves to the top of the spin-wave dispersion. We have performed calculations for disordered stripes with varying $J / J^{\prime}$ which confirmed this analysis, for details see Ref. 24 .

Turning to the charge sector, our modelling here relies on a set of parameters and assumptions [24], with rather little experimental guidance available [18]. We have tested different schemes to introduce stripe disorder, in particular resulting in a varying degree of anisotropy
$\xi_{\mathrm{C}}^{\|} / \xi_{\mathrm{C}}^{\perp}$. We have found that this has rather little influence on the magnetic behavior, such that our conclusions are independent of details of the stripe disorder [24], and the only important parameter is $\xi_{\mathrm{C}}$.

Application to $\mathrm{La}_{2-x} \mathrm{Sr}_{x} \mathrm{CoO}_{4}$. A comparison of our results with the INS data of Ref. 9 indicates that charge disorder as in Fig. 2(d) is realized in $\mathrm{La}_{5 / 3} \mathrm{Sr}_{1 / 3} \mathrm{CoO}_{4}$. For these parameters, we show constant-energy cuts of $\chi^{\prime \prime}(\vec{q}, \omega)$ in Fig. 3. We notice that the low-energy inelastic response $(\omega=0.12 J S)$ displays an anisotropic broadening - similar to the broadening of the Bragg signal, Fig. S5(a) [24], where $\xi_{\mathrm{S}}^{\|} / \xi_{\mathrm{S}}^{\perp} \approx 2.3-$ a behavior which is generic for our model. Significant low-energy intensity occurs also at $(\pi, \pi)$, a feature that, in our calculations, is generically linked to an hourglass spectrum.

For $\mathrm{La}_{5 / 3} \mathrm{Sr}_{1 / 3} \mathrm{CoO}_{4}$, we deduce $J S \approx 25 \mathrm{meV}$, for which we show an explicit comparison with the experimental data in Fig. 4. We find excellent agreement, apart from an intensity mismatch at $3 \mathrm{meV}$ which is likely due
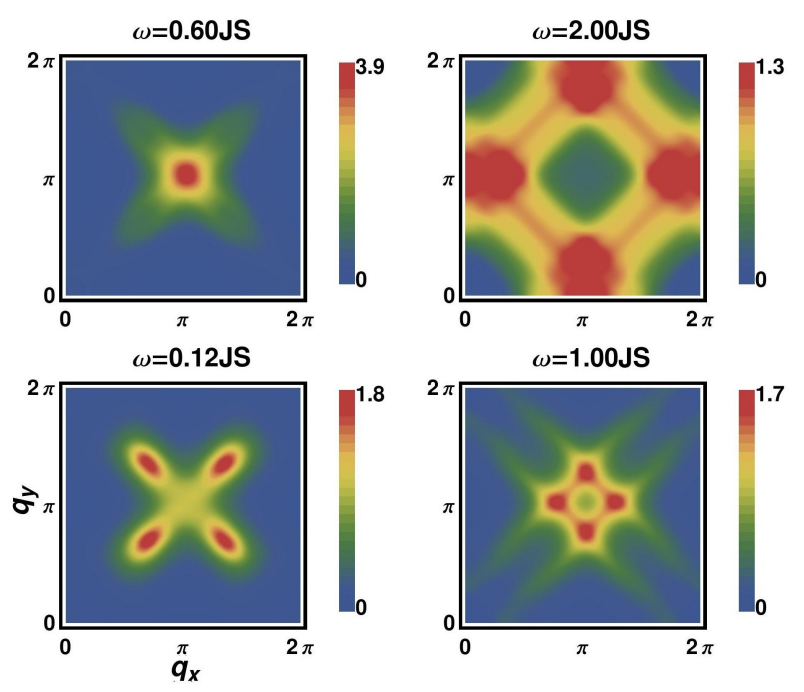

Figure 3: Constant-energy cuts of $\chi^{\prime \prime}(\vec{q}, \omega)$ with model parameters as in Fig. $2(\mathrm{~d}): J^{\prime}=0.05 J, \xi_{\mathrm{C}}^{\|} \simeq 5, \xi_{\mathrm{C}}^{\perp} \simeq 2, L=36$. 


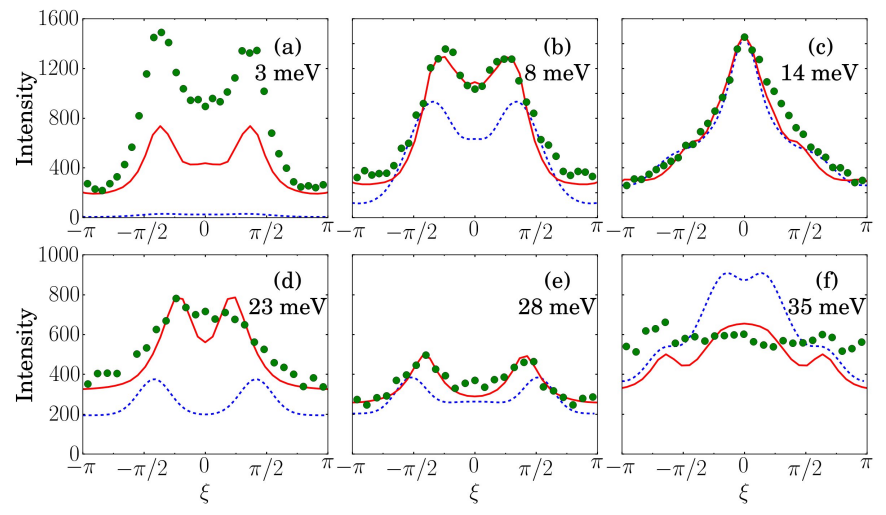

Figure 4: Comparison of $\chi^{\prime \prime}(\vec{q}, \omega)$ for parameters as in Fig. 2(d) and $J S=25 \mathrm{meV}$ (solid) to the $\mathrm{La}_{5 / 3} \mathrm{Sr}_{1 / 3} \mathrm{CoO}_{4}$ data (dots) taken from Fig. 3 of Ref. 9. Panels (a-c): $\vec{q}=(\pi+\xi, \pi-\xi) ;(\mathrm{d}-\mathrm{f}): \vec{q}=(\pi, \pi+\xi)$. Also shown are ad-hoc broadened perfect-stripe spectra (dashed) following Refs. 9, 24, with $J S=18 \mathrm{meV}$ adjusted to match the energy $\omega_{\mathrm{S}}$ of the saddle point. For both theories, the energy broadening was $\Gamma=1 \mathrm{meV}$, and an overall intensity factor and a constant background were adjusted by fitting the $14 \mathrm{meV}$ data and then were used for all $\omega[29]$.

to the simplified treatment of spin anisotropies in our spin- $1 / 2$ model [24]. Fig. 4 also shows the result of adhoc momentum-space broadening of perfect-stripe spectra [24], as used in previous modelling of the data [9]. The most obvious failure of this approach concerns the missing intensity at energies below and slightly above the saddle point; it also overestimates the intensity of the upper spin-wave band which becomes visible near $(\pi, \pi)$ at $35 \mathrm{meV}$. In addition, the mismatch of peak positions at elevated energies, Fig. 4(d,e), illustrates that the real hourglass has approximately linearly dispersing branches above the saddle point, while the perfect-stripe case yields a parabolic dispersion - a difference reproduced by our theory which becomes clear upon comparing panels (a) and (d) of Fig. 2.

We conclude that the charge stripes in $\mathrm{La}_{5 / 3} \mathrm{Sr}_{1 / 3} \mathrm{CoO}_{4}$ are rather disordered, with an estimated correlation length of $\xi_{\mathrm{C}}^{\|} \approx 5$; this explains why charge order has not been observed in scattering experiments. Our analysis predicts the magnetism in $\mathrm{La}_{5 / 3} \mathrm{Sr}_{1 / 3} \mathrm{CoO}_{4}$ to be that of a cluster spin glass. Indeed, initial signatures for glassy behavior have been detected [30], and a more thorough characterization is called for.

Finally, we propose that disordered stripes are also present in $\mathrm{La}_{2-x} \mathrm{Sr}_{x} \mathrm{CoO}_{4}$ at $x \leq 0.3$ : Smaller $x$ implies larger stripe spacing, with increased sensitivity to quenched disorder. Indeed, both a broad Bragg signal [17] and broad low-energy excitations [31] have been detected in $\mathrm{La}_{1.7} \mathrm{Sr}_{0.3} \mathrm{CoO}_{4}$ around $(\pi, \pi)$, consistent with the magnetism of strongly disordered stripes.

Other transition-metal oxides. For overdoped singlelayer manganites with ordering wavevector $\vec{Q}_{\mathrm{S}}=$
$(2 \pi \pm \delta, \pi \pm \delta)$ with $\delta=0.166 \pi$, INS measurements have recently detected hourglass spectra, in particular at elevated temperatures (while low- $T$ spectra displayed outward dispersing branches) [10]. The hourglass has been linked to a short magnetic correlation length in this regime. Our model calculations qualitatively confirm this interpretation: For intermediate $J / J^{\prime}$, the coupling between AF domains is robust at low $T$, but weakened at elevated $T$, such that the advertised hourglass excitation spectrum of weakly correlated AF clusters is observed.

Finally, it is interesting to link our study to cuprate superconductors. In fact, the physics of disordered stripes, either slowly fluctuating or disorder-pinned, has been invoked to explain a variety of experimental observations including INS data $[14,15]$. The hourglass spectra observed in cuprates show a clear absence of outward dispersing low-energy branches, and moreover display a pronounced piece of vertical dispersion in the so-called pseudogap regime $[2,3,6,15]$. Assuming that our modelling qualitatively applies to cuprates as well, we can link these experimental results to strongly disordered stripes. We also note that very underdoped (but still superconducting) cuprates have been found to display a quasieleastic "central mode" at $(\pi, \pi)$ which has been interpreted in terms of glassy short-range order [32]. Considering that decreasing the doping level in cuprates below $1 / 8$ weakens stripe order (such that it is more susceptible to quenched disorder), this nicely matches our results: For strong stripe disorder, $S_{\mathrm{S}}(\vec{q})$ looses its IC structure and instead displays a broad peak at $(\pi, \pi)$ [24].

Summary. Our detailed modelling of disordered stripes in $\mathrm{La}_{5 / 3} \mathrm{Sr}_{1 / 3} \mathrm{CoO}_{4}$ reveals a link between the celebrated hourglass excitation spectrum and a cluster-spin-glass magnetic ground state, caused by frustrated couplings between locally ordered AF domains. These phenomena appear common to a broad class of doped oxides.

We thank A. Boothroyd, M. Braden, and L. H. Tjeng for illuminating discussions, and A. Boothroyd for providing the data of Ref. 9. This research was supported by the DFG through FOR 960 and GRK 1621.

[1] M. Arai, T. Nishijima, Y. Endoh, T. Egami, S. Tajima, K. Tomimoto, Y. Shiohara, M. Takahashi, A. Garrett, and S. M. Bennington, Phys. Rev. Lett. 83, 608 (1999).

[2] J. M. Tranquada, H. Woo, T. G. Perring, H. Goka, G. D. Gu, G. Xu, M. Fujita, and K. Yamada, Nature 429, 534 (2004).

[3] S. M Hayden, H. A. Mook, P. Dai, T. G. Perring, and F. Doğan, Nature 429, 531 (2004).

[4] N. B. Christensen, D. F. McMorrow, H. M. Rønnow, B. Lake, S. M. Hayden, G. Aeppli, T. G. Perring, M. Mangkorntong, M. Nohara, and H. Takagi, Phys. Rev. Lett. 93, 147002 (2004).

[5] C. Stock, W. J. L. Buyers, R. Liang, D. Peets, Z. Tun, D. Bonn, W. N. Hardy, and R. J. Birgeneau, Phys. Rev. 
B 69, 014502 (2004).

[6] V. Hinkov, P. Bourges, S. Pailhès, Y. Sidis, A. Ivanov, C. D. Frost, T. G. Perring, C. T. Lin, D. P. Chen, and B. Keimer, Nature Phys. 3, 780 (2007).

[7] D. Reznik, J.-P. Ismer, I. Eremin, L. Pintschovius, T. Wolf, M. Arai, Y. Endoh, T. Masui, and S. Tajima, Phys. Rev. B 78, 132503 (2008).

[8] G. Xu, G. D. Gu, M. Hücker, B. Fauqué, T. G. Perring, L. P. Regnault, and J. M. Tranquada, Nature Phys. 5, 649 (2009).

[9] A. T. Boothroyd, P. Babkevich, D. Prabhakaran, and P. G. Freeman, Nature 471, 341 (2011).

[10] H. Ulbrich, P. Steffens, D. Lamago, Y. Sidis, and M. Braden, Phys. Rev. Lett. 108, 247209 (2012).

[11] I. Eremin, D. K. Morr, A. V. Chubukov, K. H. Bennemann, and M. R. Norman, Phys. Rev. Lett. 94, 147001 (2005).

[12] M. Vojta, T. Vojta, and R. K. Kaul, Phys. Rev. Lett. 97, 097001 (2006).

[13] B. M. Andersen, S. Graser, and P. J. Hirschfeld, Phys. Rev. Lett. 105, 147002 (2010).

[14] S. A. Kivelson, I. P. Bindloss, E. Fradkin, V. Oganesyan, J. M. Tranquada, A. Kapitulnik, and C. Howald, Rev. Mod. Phys. 75, 1201 (2003).

[15] M. Vojta, Adv. Phys. 58, 699 (2009).

[16] H. Ulbrich and M. Braden, preprint arXiv:1201.2356.

[17] M. Cwik, M. Benomar, T. Finger, Y. Sidis, D. Senff, M. Reuther, T. Lorenz, and M. Braden, Phys. Rev. Lett. 102, 057201 (2009).

[18] To our knowledge, detailed studies of the charge order in $\mathrm{La}_{2-x} \mathrm{Sr}_{x} \mathrm{CoO}_{4}$ are missing to date.

[19] N. Hollmann, M. W. Haverkort, M. Cwik, M. Benomar, M. Reuther, A. Tanaka, and T. Lorenz, New J. Phys. 10, 023018 (2008).

[20] P. Babkevich, D. Prabhakaran, C. D. Frost, and A. T. Boothroyd, Phys. Rev. B 82, 184425 (2010).
[21] L. M. Helme, A. T. Boothroyd, R. Coldea, D. Prabhakaran, C. D. Frost, D. A. Keen, L. P. Regnault, P. G. Freeman, M. Enderle, and J. Kulda, Phys. Rev. B 80, 134414 (2009).

[22] A. Maignan, V. Caignaert, B. Raveau, D. Khomskii, and G. Sawatzky, Phys. Rev. Lett. 93, 026401 (2004).

[23] C. F. Chang, Z. Hu, H. Wu, T. Burnus, N. Hollmann, M. Benomar, T. Lorenz, A. Tanaka, H.-J. Lin, H. H. Hsieh, C. T. Chen, and L. H. Tjeng, Phys. Rev. Lett. 102, 116401 (2009).

[24] See Supplemental Material for a detailed description of our numerical approach, additional results for the spin dynamics, a characterization of glassy behavior, and further comparison with ad-hoc broadened excitation spectra of perfect stripes.

[25] L. W. Lee and A. P. Young, Phys. Rev. B 76, 024405 (2007); D. X. Viet and H. Kawamura, ibid. 80, 064418 (2009).

[26] A. T. Boothroyd, D. Prabhakaran, P. G. Freeman, S. J. S. Lister, M. Enderle, A. Hiess, and J. Kulda, Phys. Rev. B 67, 100407(R) (2003).

[27] H. Woo, A. T. Boothroyd, K. Nakajima, T. G. Perring, C. D. Frost, P. G. Freeman, D. Prabhakaran, K. Yamada, and J. M. Tranquada, Phys. Rev. B 72, 064437 (2005).

[28] D. X. Yao, E. W. Carlson, and D. K. Campbell, Phys. Rev. B 73, 224525 (2006).

[29] The experimental data for $\omega>14 \mathrm{meV}$ in Fig. 3 of Ref. 9 were taken under conditions different from that for the low- $\omega$ data, such that the intensity scales are not strictly comparable [30].

[30] A. T. Boothroyd, private communication.

[31] M. Cwik, PhD thesis, Universität zu Köln (2007).

[32] C. Stock, W. J. L. Buyers, Z. Yamani, C. L. Broholm, J.-H. Chung, Z. Tun, R. Liang, D. Bonn, W. N. Hardy, and R. J. Birgeneau, Phys. Rev. B 73, 100504 (2006). 


\title{
Supplementary information for: "Disorder, cluster spin glass, and hourglass spectra in striped magnetic insulators"
}

\author{
Eric C. Andrade and Matthias Vojta \\ Institut für Theoretische Physik, Technische Universität Dresden, 01062 Dresden, Germany
}

(Dated: November 9, 2018)

\section{GENERATING CHARGE CONFIGURATIONS}

The charge order of $\mathrm{Co}^{2+}$ and $\mathrm{Co}^{3+}$ ions governs the magnetism of $\mathrm{La}_{2-x} \mathrm{Sr}_{x} \mathrm{CoO}_{4}$. As this charge order sets in above room temperature, we assume the charge order to be static on the time scale of magnetic fluctuations, such that the spin sector can be analyzed - in the spirit of a Born-Oppenheimer approximation - for a set of frozen charge configurations. Those configurations correspond to imperfect stripes, with imperfections likely caused by crystalline defects.

We obtain such charge configurations from numerical simulations of an appropriate Ising model. Consider variables $n_{i}$ on the sites $i$ of a square lattice, where $n_{i}=0$ refers to a $\mathrm{Co}^{2+}$ ion, while $n_{i}=0$ refers to a $\mathrm{Co}^{3+}$ ion. The $n_{i}$ are subject to a Hamiltonian of the form

$$
\mathcal{H}_{C}=\sum_{m=1}^{5} V_{m} \sum_{\langle i, j\rangle_{m}} n_{i} n_{j}
$$

with a particle density of $\sum_{i} n_{i}=N / 3$ adequate to describe the compound $\mathrm{La}_{5 / 3} \mathrm{Sr}_{1 / 3} \mathrm{CoO}_{4}$, where $N=L \times L$ is the number of sites, and periodic boundary conditions are applied. The $V_{m}$ represent density-density interactions as depicted in Fig. S1, which are chosen such that the ground state of $\mathcal{H}_{C}$ is given by the diagonal stripe state established for $\mathrm{La}_{5 / 3} \mathrm{Sr}_{1 / 3} \mathrm{CoO}_{4}$, Fig. S1. Specifically, $V_{1}$ is a nearest-neighbor repulsion, $V_{2}<0$ a secondneighbor attraction, and $V_{3}>0$ a third-neighbor repulsion, while $V_{4,5}$ are longer-range interaction used to modify the defect structure (see below, for most of the calculations $V_{4,5}=0$ ).

We generate short-range-ordered charge configurations using Monte-Carlo (MC) simulations of the Ising model in Eq. (S1). As noted above, the relevant charge disorder is not generated by thermal fluctuations at temperatures near or above a putative charge-ordering transition (because this temperature is far above room temperature in $\mathrm{La}_{2-x} \mathrm{Sr}_{x} \mathrm{CoO}_{4}$ ), but instead arises from structural defects (e.g. the dopant distribution). As details of this quenched disorder are not known, and in order to keep the set of input parameters minimal, we choose to employ the model Eq. (S1) without quenched disorder, but instead extract configurations away from thermal equilibrium.

To this end, the variables $\left\{n_{i}\right\}$ are initialized by randomly distributing $N / 3$ charges on $N$ sites. One MC step consists of randomly selecting two sites and exchanging their charges, accepting or rejecting this move according (a)

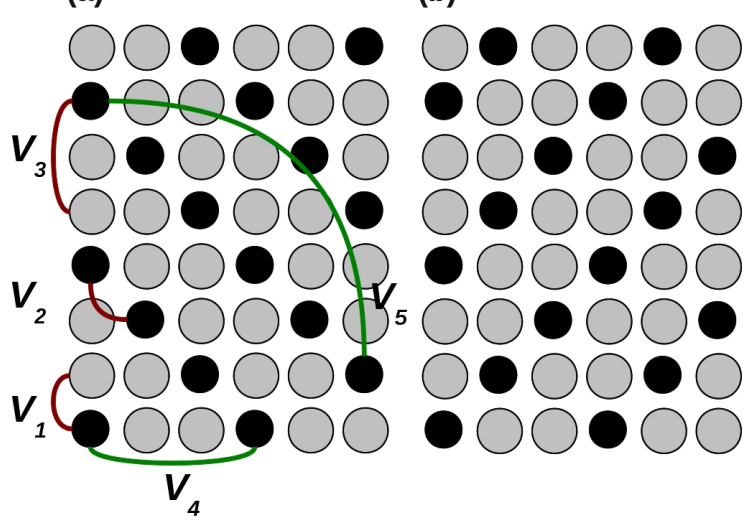

Figure S1: Perfect diagonal period-3 stripe patterns in the $\mathrm{CoO}_{2}$ planes of $\mathrm{La}_{5 / 3} \mathrm{Sr}_{1 / 3} \mathrm{CoO}_{4}$, where black and gray circles correspond to $n_{i}=1\left(\mathrm{Co}^{3+}\right)$ and $n_{i}=0\left(\mathrm{Co}^{2+}\right)$, respectively. These configurations are ground states of the Ising model in Eq. (S1), with density-density interactions $V_{1}>0, V_{2}<0$ and $V_{3}>0$ as shown in panel a). The longer-range interactions $V_{4,5}$ will be utilized to modify the structure of charge-order defects away from the ground state.

to the rules of the standard Metropolis algorithm at a finite effective temperature $T$. We work at a low temperature corresponding to the charge-ordered phase and select configurations $\left\{n_{i}\right\}$ after a fixed number $N_{\mathrm{MC}}$ of MC steps. Thus, for large $N_{\mathrm{MC}}$ the system reaches a state with long-range order, while smaller $N_{\mathrm{MC}}$ result in short-range-ordered states. We assume that these states sensibly represent the situation in $\mathrm{La}_{2-x} \mathrm{Sr}_{x} \mathrm{CuO}_{4}$, because working at low $T$ ensures to have well-ordered regions separated by localized defect structures, as can be expected for a material far below its charge-ordering temperature. Observables are finally obtained by averaging over a large number of charge configurations, all obtained from runs with different initial conditions - this mimics the average over a large disordered sample.

To quantify the charge disorder, we calculate the charge correlation length $\xi_{\mathrm{C}}$, which we obtain from the static charge structure factor

$$
S_{\mathrm{C}}(\vec{q})=\frac{1}{N_{\mathrm{S}}}\left[\sum_{i, j}\left(1-n_{i}\right)\left(1-n_{j}\right) e^{-i \vec{q} \cdot \vec{r}_{i j}}\right]_{a v},
$$

where $N_{\mathrm{S}}=2 N / 3$ is the number of $\mathrm{Co}^{2+}$ ions (i.e. spins), and $[\cdots]_{a v}$ denotes the average over charge configurations. For short-range order, $S_{\mathrm{C}}(\vec{q})$ is typically peaked at four wavevectors $\vec{Q}_{\mathrm{C}}$ (see Fig. 1(d) of the main paper and 


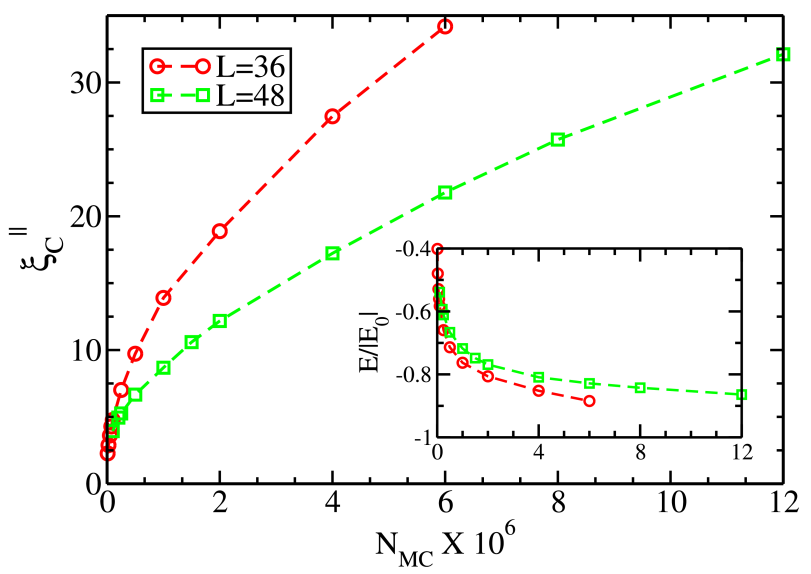

Figure S2: Evolution of the charge correlation length $\xi_{\mathrm{C}}$ with $N_{\mathrm{MC}}$, i.e., along the MC simulation of the Ising model Eq. (S1) for two different system sizes $L$. The parameters $V_{1}=4, V_{2}=-2, V_{3}=4, V_{4,5}=0$ and $T=1$ correspond to the charge-ordered phase, such that $\xi_{\mathrm{C}}$ reaches the system size for large $N_{\mathrm{MC}}$. The inset shows the evolution of the energy $E=\left[\mathcal{H}_{C}\right]_{a v}$ along the MC simulation, normalized to the energy of the perfectly ordered state $E_{0}$.

Figs. S4, S6): the peaks at $\vec{Q}_{\mathrm{C}}=(2 \pi / 3,2 \pi / 3)$ and $\vec{Q}_{\mathrm{C}}=$ $(4 \pi / 3,4 \pi / 3)$ correspond to stripe domains like those in Fig. S1(a), while peaks at $\vec{Q}_{\mathrm{C}}=(2 \pi / 3,4 \pi / 3)$ and $\vec{Q}_{\mathrm{C}}=(4 \pi / 3,2 \pi / 3)$ belong to stripes running along the opposite diagonal, Fig. S1(b). The width of the peaks is anisotropic, thus we distinguish correlation lengths $\xi_{\mathrm{C}}^{\|}$ and $\xi_{\mathrm{C}}^{\perp}$ parallel and perpendicular to the stripe direction, respectively. The evolution of $\xi_{\mathrm{C}}$ with the number of steps, $N_{\mathrm{MC}}$, within our non-equilibrium MC procedure is shown in Fig. S2. As anticipated, the ordered state (with $\xi_{\mathrm{C}} \gtrsim L$ ) is reached for large $N_{\mathrm{MC}}$, while smaller values, $N_{\mathrm{MC}}=10^{4}-10^{6}$, are suitable for obtaining charge configurations with $\xi_{\mathrm{C}} \approx 2-10$ lattice spacings.

Individual charge configuration from our MC simulations are shown in the top rows of Figs. S4(b)-(e) and S6. We see that the protocol generates finite-size stripe domains of both orientations, as intended. For a large range of parameters with $V_{4,5}=0$ as in Fig. S4 we generically find $\xi_{\mathrm{C}}^{\|}>\xi_{\mathrm{C}}^{\perp}$, i.e., the diagonal arrangement of neighboring charges tends to be more stable than the spacing between the diagonal charge lines. $S_{\mathrm{C}}(\vec{q})$ also reveals that for very large disorder, Fig. S4(e), significant weight appears away from $\vec{Q}_{\mathrm{C}}$. This effect is even more pronounced in the spin sector, as will be discussed below.

Charge configurations with $\xi_{\mathrm{C}}^{\|}<\xi_{\mathrm{C}}^{\perp}$ can be generated on purpose, e.g., by choosing the longer-range couplings $V_{4,5} \neq 0$, which stabilize the stripe spacing and cut charge lines, respectively. Results are shown in Fig. S6, which now show a visibly different defect structure in the charge sector. Remarkably, the behavior on the spin sector is rather insensitive to this change (see below).

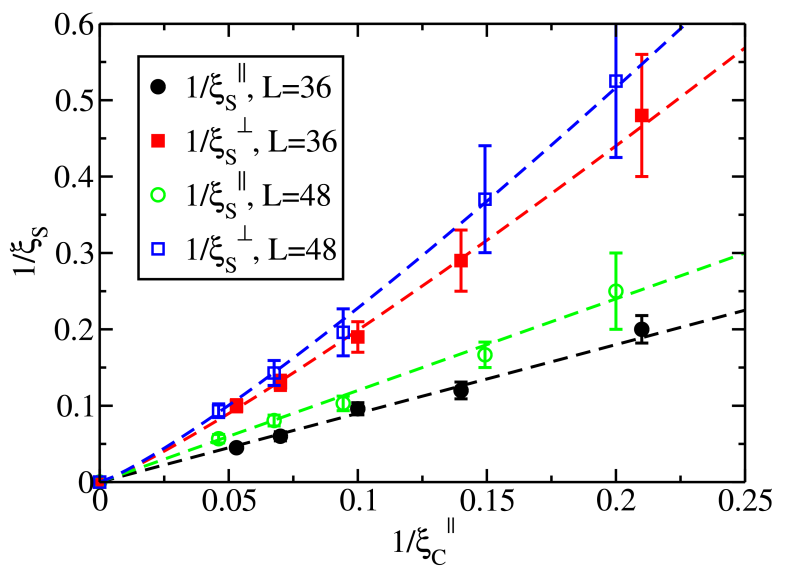

Figure S3: Spin correlation lengths, $\xi_{\mathrm{S}}^{\perp}$ and $\xi_{\mathrm{S}}^{\|}$, of the classical ground state of $\mathcal{H}_{\mathrm{sp}}$, Eq. (S3), as function of the charge correlation length $\xi_{\mathrm{C}}^{\|}$which characterized the stripe disorder. Clearly, $\xi_{\mathrm{S}}^{\|} \approx \xi_{\mathrm{C}}^{\|}$while $\xi_{\mathrm{S}}^{\perp}$ is smaller by a factor of $2-2.5$.

\section{SPIN MODEL}

As explained in the main text, the magnetism of $\mathrm{La}_{5 / 3} \mathrm{Sr}_{1 / 3} \mathrm{CoO}_{4}$ is assumed to be well described by immobile magnetic moments that are located on the $\mathrm{Co}^{2+}$ sites (i.e. those with $n_{i}=0$ ) of a disordered 2D stripe configuration.

The $\mathrm{Co}^{2+}$ ions are in a $3 \mathrm{~d}^{7}$ high-spin configuration with $S=3 / 2$. A full modelling of these high-spin ions and their interactions has been put forward in Refs. 1,2 - this involves spin and orbital angular momentum, crystalfield and spin-orbit effects, as well as (nearly isotropic) exchange interactions. As the local Hilbert space in this model contains 28 states, it is more efficient to employ a reduced effective model instead: As shown in Refs. 1,2 , the low-energy part of the excitation spectrum of $\mathrm{La}_{2-x} \mathrm{Sr}_{x} \mathrm{CuO}_{4}$ both at $x=0$ and $x=0.5$ can be well captured by a $2 \mathrm{D}$ spin- $1 / 2$ spin-only model.

Consequently, we adopt such a model for our purpose,

$$
\mathcal{H}_{\mathrm{sp}}=\sum_{i, j} \sum_{\alpha} J_{i j}^{\alpha} S_{i}^{\alpha} S_{j}^{\alpha}
$$

and choose the nearest-neighbor $J$ as in Refs. 1,2 to be $J^{x}=J(1+\epsilon), J^{y}=J$, and $J^{z}=J(1-\delta)$, with $\delta=0.28$ and $\epsilon=0.013$. This exchange anisotropy mimics the effect of the original single-ion anisotropy, resulting from the combination of crystal-field and spin-orbit interactions. It is chosen such that the ordered AF state has spins in the $x$ direction, corresponding to the crystallographic a axis, as in experiment. Following Ref. 3 we neglect the anisotropy of the smaller coupling $J^{\prime}$ across a non-magnetic $\mathrm{Co}^{3+}$ ion.

As discussed in the main text, for imperfect charge order, the classical ground state of $\mathcal{H}_{\mathrm{sp}}$ is a cluster spin glass with well-defined short-range magnetic order, see 
also Sec. VI below. In Fig. S3, we demonstrate the correlation between $\xi_{\mathrm{S}}$ and $\xi_{\mathrm{C}}$. In particular, we obtain $\xi_{\mathrm{S}}^{\|} \approx \xi_{\mathrm{C}}^{\|}$for the system sizes considered.

\section{SPIN-WAVE CALCULATION}

We calculate the excitation spectrum of $\mathcal{H}_{\mathrm{sp}}$ (which involves spatial disorder) using real-space linear spin-wave theory $^{5-7}$ on finite $L \times L$ lattices with periodic boundary conditions. This is done by expanding around a locally stable classical state (or saddle point) of $\mathcal{H}_{\mathrm{sp}}$ as determined by the spin MC procedure described in the main text. The relevant classical states are in general noncollinear, with the spin directions of the $\vec{S}_{i}$ characterized by angles $\theta_{i}$ and $\phi_{i}$. We start by introducing a new reference frame in which $S_{i}^{z \prime}$ points locally in the direction of the magnetization

$$
\left(\begin{array}{c}
S_{i}^{x} \\
S_{i}^{y} \\
S_{i}^{z}
\end{array}\right)=\left(\begin{array}{ccc}
\cos \theta_{i} \cos \phi_{i} & -\sin \phi_{i} & \sin \theta_{i} \cos \phi_{i} \\
\cos \theta_{i} \sin \phi_{i} & \cos \phi_{i} & \sin \theta_{i} \sin \phi_{i} \\
-\sin \theta_{i} & 0 & \cos \theta_{i}
\end{array}\right)\left(\begin{array}{c}
S_{i}^{x \prime} \\
S_{i}^{y \prime} \\
S_{i}^{z \prime}
\end{array}\right)
$$

In this rotated frame $\vec{S}^{\prime}$, we apply the linearized HolsteinPrimakoff transformation: $S_{i}^{+\prime}=\sqrt{2 S_{i}} a_{i}, \quad S_{i}^{-\prime}=$ $\sqrt{2 S_{i}} a_{i}^{\dagger}$, and $S_{i}^{z \prime}=S_{i}-a_{i}^{\dagger} a_{i}$, where $S_{i}=1 / 2$ is the spin size at site $i$. The bilinear piece $\mathcal{H}_{2}$ of the Hamiltonian then reads

$$
\mathcal{H}_{2}=\sum_{i j} A_{i j} a_{i}^{\dagger} a_{j}+\frac{1}{2} \sum_{i j}\left(B_{i j} a_{i}^{\dagger} a_{j}^{\dagger}+\text { h.c. }\right),
$$

with the coefficients

$$
\begin{aligned}
A_{i j} & =-2 \delta_{i j} \sum_{k} F_{i k}^{z z} S_{k}+\sqrt{S_{i}} \sqrt{S_{j}}\left[F_{i j}^{x x}+F_{i j}^{y y}-\right. \\
& \left.-i\left(F_{i j}^{x y}-F_{j i}^{x y}\right)\right] \\
B_{i j} & =\sqrt{S_{i}} \sqrt{S_{j}}\left[F_{i j}^{x x}-F_{i j}^{y y}+i\left(F_{i j}^{x y}+F_{j i}^{x y}\right)\right] .
\end{aligned}
$$

Here, the functions $F_{i j}^{\alpha \beta}$ read

$$
\begin{aligned}
F_{i j}^{x x} & =J_{i j}^{x} \cos \theta_{i} \cos \theta_{j} \cos \phi_{i} \cos \phi_{j}+ \\
& +J_{i j}^{y} \cos \theta_{i} \cos \theta_{j} \sin \phi_{i} \sin \phi_{j}+J_{i j}^{z} \sin \theta_{i} \sin \theta_{j} \\
F_{i j}^{y y} & =J_{i j}^{x} \sin \phi_{i} \sin \phi_{j}+J_{i j}^{y} \cos \phi_{i} \cos \phi_{j} \\
F_{i j}^{z z} & =J_{i j}^{x} \sin \theta_{i} \sin \theta_{j} \cos \phi_{i} \cos \phi_{j}+ \\
& +J_{i j}^{y} \sin \theta_{i} \sin \theta_{j} \sin \phi_{i} \sin \phi_{j}+J_{i j}^{z} \cos \theta_{i} \cos \theta_{j} \\
F_{i j}^{x y} & =-J_{i j}^{x} \cos \theta_{i} \cos \phi_{i} \sin \phi_{j}+J_{i j}^{y} \cos \theta_{i} \sin \phi_{i} \cos \phi_{j} .
\end{aligned}
$$

The bilinear Hamiltonian Eq. (S4) can be numerically diagonalized using a bosonic Bogoliubov transformation, ${ }^{5-7}$ and the resulting Hamiltonian reads (up to a constant)

$$
\mathcal{H}_{2}=\sum_{\nu} \omega_{\nu} b_{\nu}^{\dagger} b_{\nu}
$$

where $\omega_{\nu}$ is the eigenfrequency associated to the $\nu$ th mode, and

$$
\left(\begin{array}{c}
a \\
a^{\dagger}
\end{array}\right)=\left(\begin{array}{cc}
U & V \\
U^{\star} & V^{\star}
\end{array}\right)\left(\begin{array}{c}
b \\
b^{\dagger}
\end{array}\right)
$$

with $a^{T}=\left(a_{1}, a_{2}, \cdots, a_{N_{\mathrm{S}}}\right), b^{T}=\left(b_{1}, b_{2}, \cdots, b_{N_{\mathrm{S}}}\right)$. $U$ and $V$ are $N_{\mathrm{S}} \times N_{\mathrm{S}}$ matrices describing the transformation to this diagonal basis. ${ }^{8,9}$

Finally, we evaluate the rotationally averaged dynamic susceptibility, $\chi^{\prime \prime}(\vec{q}, \omega)$, in the standard one-magnon approximation, see Eq. (2) of the main paper. For a single realization of disorder and using the spin-wave transformation as described above we have

$$
\chi^{\prime \prime}(\vec{q}, \omega)=\sum_{\nu} \sum_{\alpha}\left|M_{\nu}^{\alpha}(\vec{q})\right|^{2} \delta\left(\omega-\omega_{\nu}\right) .
$$

Here $\alpha=x, y, z$ and $M_{\nu}^{\alpha}(\vec{q})$ represents the matrix element $\left\langle 0\left|S^{\alpha}(\vec{q})\right| \nu\right\rangle$ of the Fourier-transformed spin operator with the eigenmode $|\nu\rangle$ :

$$
M_{\nu}^{\alpha}(\vec{q})=\frac{1}{\sqrt{L^{2}}} \sum_{i} e^{-i \vec{q} \cdot \vec{r}_{i}} M_{\nu}^{\alpha}\left(\vec{r}_{i}\right)
$$

where

$$
\begin{aligned}
M_{\nu}^{x}\left(\vec{r}_{i}\right) & =\sqrt{\frac{S_{i}}{2}}\left[U_{i \nu}\left(\cos \theta_{i} \cos \phi_{i}+i \sin \phi_{i}\right)+\right. \\
& \left.+V_{i \nu}^{\star}\left(\cos \theta_{i} \cos \phi_{i}-i \sin \phi_{i}\right)\right] \\
M_{\nu}^{y}\left(\vec{r}_{i}\right) & =\sqrt{\frac{S_{i}}{2}}\left[U_{i \nu}\left(\cos \theta_{i} \sin \phi_{i}-i \cos \phi_{i}\right)+\right. \\
& \left.+V_{i \nu}^{\star}\left(\cos \theta_{i} \sin \phi_{i}+i \cos \phi_{i}\right)\right] \\
M_{\nu}^{z}\left(\vec{r}_{i}\right) & =\sqrt{\frac{S_{i}}{2}}\left[-\sin \theta_{i}\left(U_{i \nu}+V_{i \nu}^{\star}\right)\right] .
\end{aligned}
$$

As momentum is not a good quantum number in a disordered system, the matrix element $M_{\nu}^{\alpha}(\vec{q})$, for any given $\vec{q}$, will in general be non-zero for all eigenmodes $\nu$.

\section{CHARGE DISORDER AND HOURGLASS SPECTRUM: ADDITIONAL RESULTS}

The evolution of the spin excitations with increasing charge disorder is shown in Fig. 2 of the main paper. Here we complement this by Fig. S4 which displays snapshots of the charge configuration as well as $S_{\mathrm{C}}(\vec{q})$ and $S_{\mathrm{S}}(\vec{q})$ for the same parameters as in Fig. 2. The figure reveals a very similar progressive and anisotropic broadening in $S_{\mathrm{C}}(\vec{q})$ and $S_{\mathrm{S}}(\vec{q})$ for weak and moderate disorder. This is different for strong disorder, Fig. S4(e), where the IC signal in $S_{\mathrm{S}}(\vec{q})$ has almost disappeared in favor of a broad peak at the $\mathrm{AF}$ wavevector $(\pi, \pi)$, while $S_{\mathrm{C}}(\vec{q})$ still displays a clear IC structure. This corresponds to a state where disorder-induced frustration has destroyed the period-3 spin correlations, leaving behind weakly correlated local AF clusters - this is further illustrated in Fig. S10 below. 

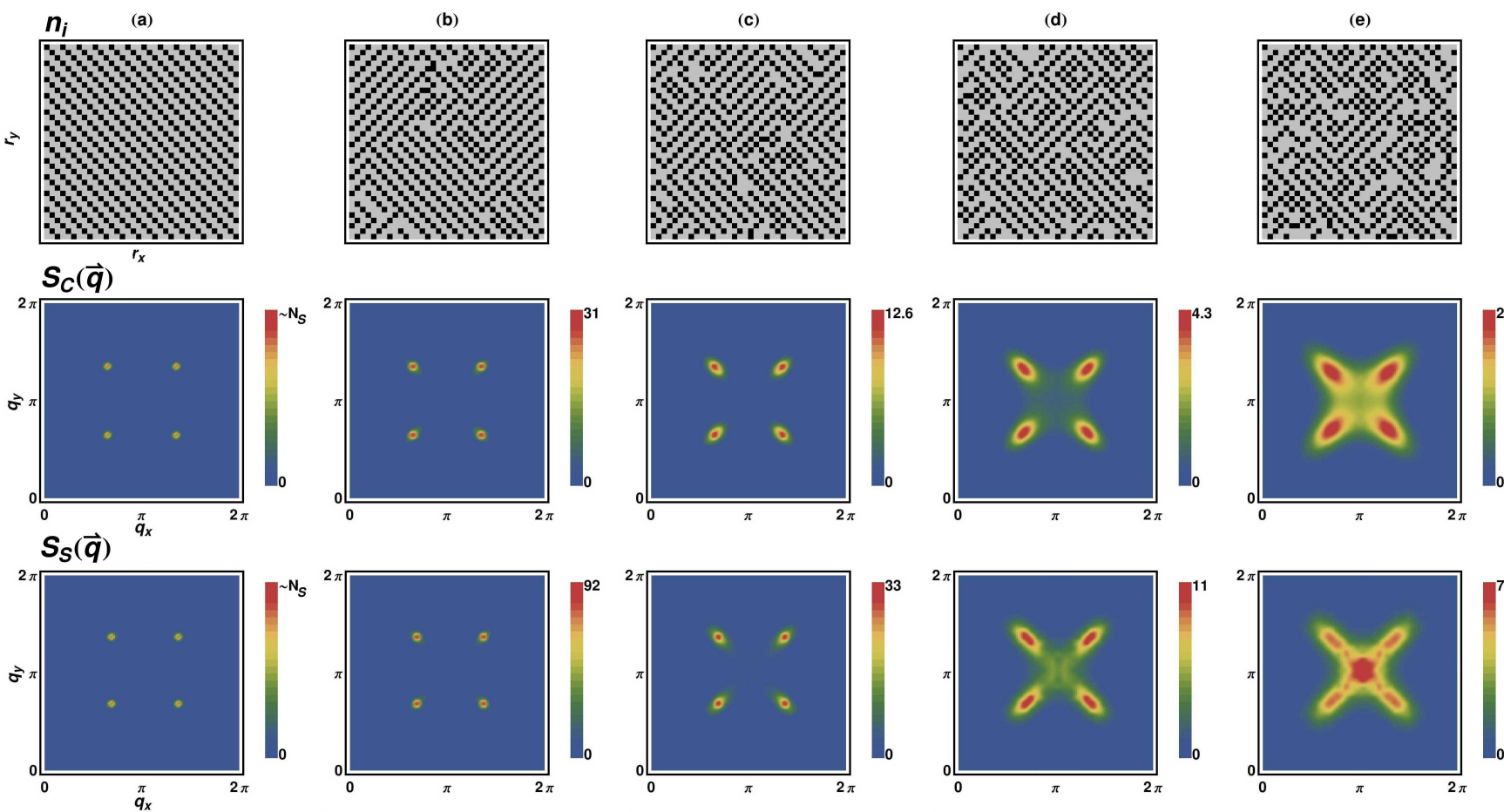

Figure S4: Numerical results for individual charge configurations $n_{i}$ (top), the charge structure factor $S_{\mathrm{C}}(\vec{q})$ (middle), and the spin structure factor $S_{\mathrm{S}}(\vec{q})$ (bottom) for disordered stripes with $L=36$. From (a) to (e), the degree of charge disorder increases as in Fig. 2 of the main paper. For the charge sector, the parameters are $V_{1}=4, V_{2}=-2, V_{3}=2, V_{4,5}=0, T=1$, and (a) perfect stripes, (b) $N_{\mathrm{MC}}=2 \times 10^{6}, \xi_{\mathrm{C}}^{\|} \simeq 19, \xi_{\mathrm{C}}^{\perp} \simeq 8$, (c) $N_{\mathrm{MC}}=0.5 \times 10^{6}, \xi_{\mathrm{C}}^{\|} \simeq 10, \xi_{\mathrm{C}}^{\perp} \simeq 4,(\mathrm{~d}) N_{\mathrm{MC}}=0.1 \times 10^{6}$, $\xi_{\mathrm{C}}^{\|} \simeq 5, \xi_{\mathrm{C}}^{\perp} \simeq 2$, (e) $N_{\mathrm{MC}}=0.025 \times 10^{6}, \xi_{\mathrm{C}}^{\|} \simeq 3, \xi_{\mathrm{C}}^{\perp} \lesssim 1$. The spin excitation spectra corresponding to the situations in panels (a) to (e) are displayed in Fig. 2 of the main paper, which also specifies the parameters of the spin model $\mathcal{H}_{\mathrm{sp}}$.

\section{A. Spin-sector parameters}
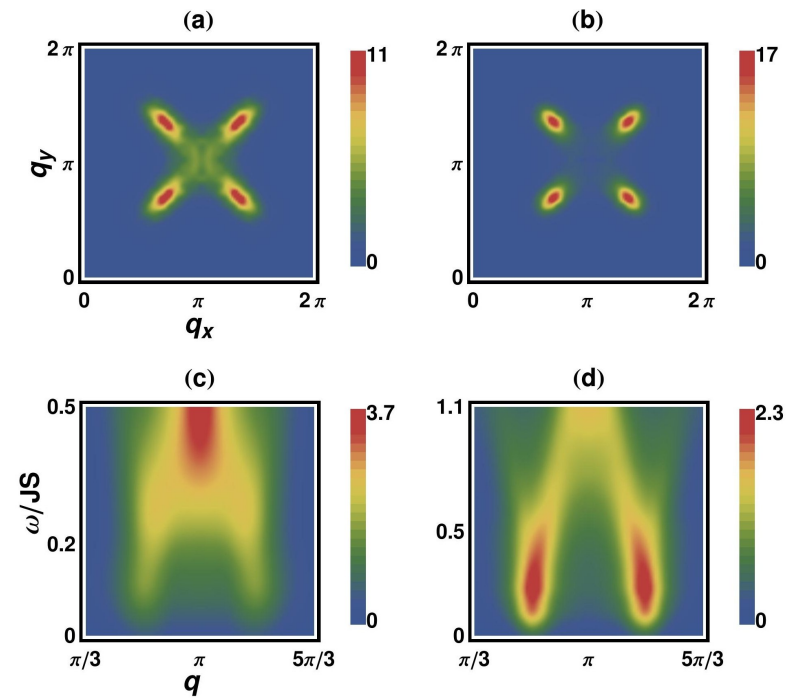

Figure S5: Influence of the coupling ratio $J / J^{\prime}$ on the magnetism, for charge-stripe parameters as in Fig. 2(d) of the main paper. (a,b) $S_{\mathrm{S}}(\vec{q})$ and (c,d) low-energy part of $\chi^{\prime \prime}(\vec{q}, \omega)$ along $\vec{q}=(q, q)$ for $(\mathrm{a}, \mathrm{c}) J^{\prime}=0.05 J$ and $(\mathrm{b}, \mathrm{d}) J^{\prime}=0.15 J$.
We have performed calculations for disordered stripes with varying $J / J^{\prime}$, with sample results in Fig. S5. These confirm that $J / J^{\prime}$ decisively influences the form of the magnetic spectrum: While an hourglass emerges for $J / J^{\prime}=20$, for $J / J^{\prime} \sim 7$ there is neither a large-intensity feature at $(\pi, \pi)$ nor a "vertical" dispersion. Instead, large weight is concentrated at low energies close to $\vec{Q}_{\mathrm{S}}$, even though the outward dispersing branches are weakened by disorder. Moreover, the structure factor $S_{\mathrm{S}}(\vec{q})$ reveals that the spin sector is less sensitive to charge disorder for smaller $J / J^{\prime}$ : stronger inter-stripe coupling stabilizes the IC state against glassy behavior.

We note that $J / J^{\prime}$ is often deduced via the experimentally measured location of the saddle point, $\omega_{\mathrm{S}} /(J S)$. Our results in Fig. 2 of the main paper show that care is required, because $\omega_{\mathrm{S}} /(J S)$ also depends on the degree of stripe disorder.

\section{B. Charge-sector parameters}

As mentioned in Sec. I, the disordered stripes obtained from our MC procedure quite generically display $\xi_{\mathrm{C}}^{\|}>\xi_{\mathrm{C}}^{\perp}$ 


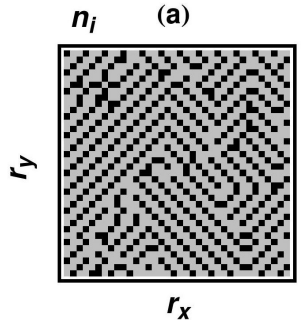

(b)

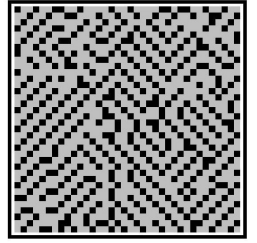

$S_{C}(\vec{q})$
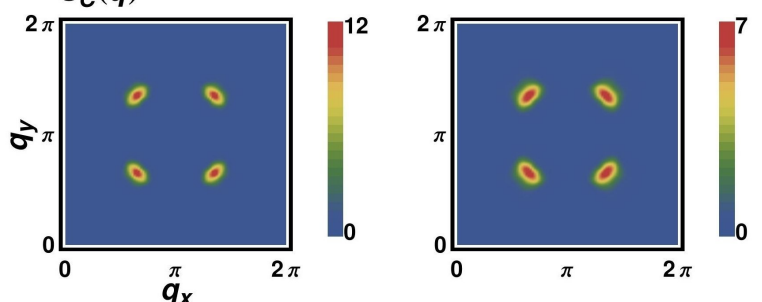

$S_{S}(\vec{q})$
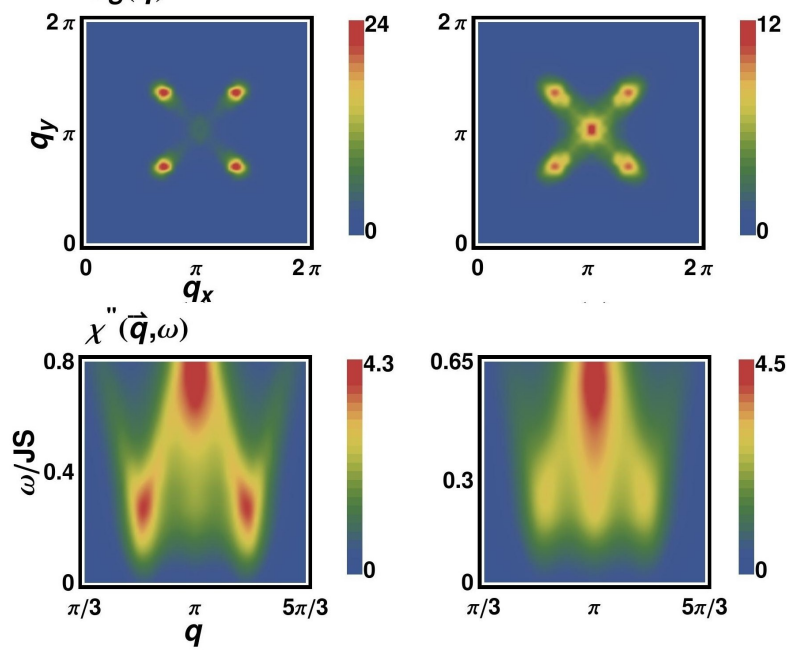

Figure S6: Numerical results for disordered stripes as in Fig. S4, but here for stripe parameters with $\xi_{\mathrm{C}}^{\|}<\xi_{\mathrm{C}}^{\perp}$. From top to bottom: charge configuration $n_{i}$, charge structure factor $S_{\mathrm{C}}(\vec{q})$, spin structure factor $S_{\mathrm{S}}(\vec{q})$, and the low-energy part of the magnetic excitations, $\chi^{\prime \prime}(\vec{q}, \omega)$, along $\vec{q}=(q, q)$. The parameters for the charge sector are $L=36, V_{1}=2, V_{2}=0$, $V_{3}=2, V_{4}=-2, V_{5}=0.5, T=1$, and (a) $N_{\mathrm{MC}}=4 \times 10^{4}$, $\xi_{\mathrm{C}}^{\|} \simeq 5, \xi_{\mathrm{C}}^{\perp} \simeq 10$, (b) $N_{\mathrm{MC}}=0.5 \times 10^{4}, \xi_{\mathrm{C}}^{\|} \simeq 2, \xi_{\mathrm{C}}^{\perp} \simeq 5$. For the spin sector we have $J / J^{\prime}=20$ and the $\delta$ peaks in $\chi^{\prime \prime}(\vec{q}, \omega)$ were replaced by Lorentzians with width $\Gamma=0.1 J S$. Remarkably, $S_{\mathrm{S}}(\vec{q})$ displays anisotropic broadening with $\xi_{\mathrm{S}}^{\|}>\xi_{\mathrm{S}}^{\perp}$, despite $\xi_{\mathrm{C}}^{\|}<\xi_{\mathrm{C}}^{\perp}$ in the charge sector. Consequently, the spin excitations here are qualitatively similar to that in Fig. 2 of the main paper (for a comparable degree of charge disorder).

- this applies to all cases in Fig. S4. Results for the opposite situation, $\xi_{\mathrm{C}}^{\|}<\xi_{\mathrm{C}}^{\perp}$, are shown in Fig. S6 - those were obtained using $V_{4,5} \neq 0$ in $\mathcal{H}_{C}$, Eq. (S1). Remarkably, even for this kind of disorder configuration we find $\xi_{\mathrm{S}}^{\|}>\xi_{\mathrm{S}}^{\perp}$ which thus appears universal. This can be rationalized considering that we work in the limit $J \gg J^{\prime}$ : Here, intact AF domains will show nearly perfect order, while correlations between AF domains, mediated by the small $J^{\prime}$, can easily be destroyed by spatial disorder. As the geometry implies that $J$ stabilizes correlations along the stripe direction, this explains $\xi_{\mathrm{S}}^{\|}>\xi_{\mathrm{S}}^{\perp}$. It is then not surprising that the evolution of the spin excitation spectrum in the case $\xi_{\mathrm{C}}^{\|}<\xi_{\mathrm{C}}^{\perp}$, shown in the bottom row of Fig. S6, in qualitatively similar to that in the case $\xi_{\mathrm{C}}^{\|}>\xi_{\mathrm{C}}^{\perp}$, Fig. 2 of the main paper: While both highintensity features at $\vec{Q}_{\mathrm{S}}$ and outward dispersing branches at low energy are still visible for weak disorder, those tend to be suppressed for strong disorder, where instead an apparent "vertical" dispersion appears at intermediate energies.

An interesting feature in Fig. S6(b) is a considerable enhancement of the intensity at $(\pi, \pi)$ even for energies well below the saddle point, coexisting with lowenergy weight near $\vec{Q}_{\mathrm{S}}$. This can be traced back to the momentum-space structure of $S_{\mathrm{S}}(\vec{q})$, which shows peaks both at $\vec{Q}_{\mathrm{S}}$ and $(\pi, \pi)$, arising from the coexistence of relatively well-defined period-3 structures and small AF clusters. (We have not observed this pronounced coexistence for disordered stripes with $\xi_{\mathrm{C}}^{\|}>\xi_{\mathrm{C}}^{\perp}$, implying that microscopic details of the charge sector play some role here.) A further increase of the charge disorder tends to transfer spectral weight from $\vec{Q}_{\mathrm{S}}$ to $(\pi, \pi)$, similar to Fig. 2(e) of the main paper.

\section{COMPARISON TO BROADENED PERFECT-STRIPE SPECTRA}

In order to further illustrate the importance of a proper treatment of stripe disorder, we provide a comparison of our results to those from spin-wave calculations for perfectly ordered stripes, where disorder is simply accounted for by an ad-hoc broadening in momentum space. This procedure has been employed previously in the literature: For instance, in Ref. 3 reasonable agreement with the experimental data concerning the intensity distribution in momentum space was achieved.

We have implemented the ad-hoc broadening for the spin-1/2 model in Eq. (S3) following Ref. 3, by convolving $\chi^{\prime \prime}(\vec{q}, \omega)$ at a fixed energy $\omega$ with a two-dimensional anisotropic Gaussian. This introduces two fit parameters - the momentum-space widths $\gamma_{\|}$and $\gamma_{\perp}$ - which mimick stripe disorder and thus replace the physical parameter $\xi_{\mathrm{C}}$ of our calculation. Results for broadened perfectstripe spectra with $J / J^{\prime}=20$ are shown in Fig. S7, where $\gamma_{\|}$and $\gamma_{\perp}$ were chosen as to match the momentum width of the experimentally observed excitations at low energy ( $3 \mathrm{meV}$ in Fig. 4 of Ref. 3). (The same parameters are used for the dashed curves in Fig. 4 of the main text.) As a result, the momentum-space intensity distributions are roughly similar to that obtained from the full calculation displayed in Fig. 3 of the main paper (and also roughly similar to that of the experimental data in Fig. 4 of Ref. 3). 

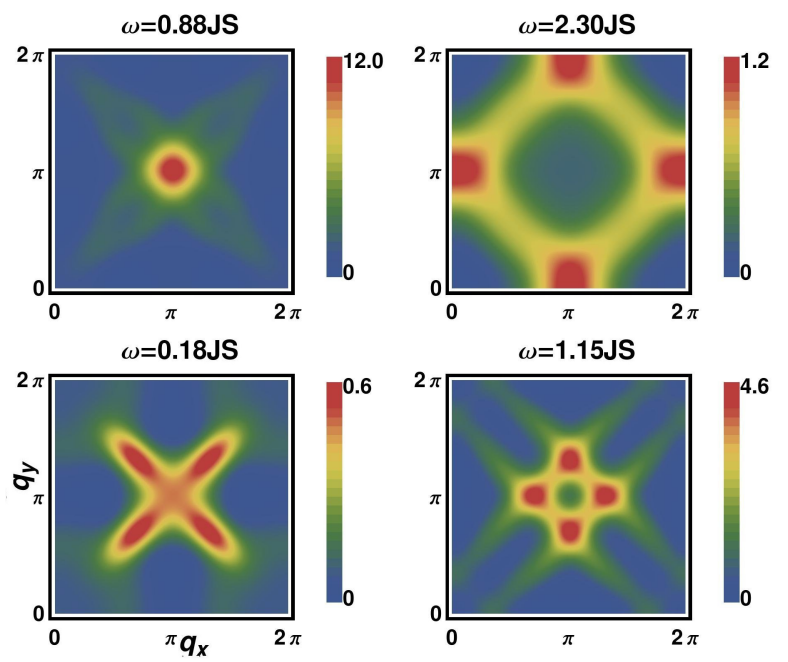

Figure S7: Constant-energy cuts of $\chi^{\prime \prime}(\vec{q}, \omega)$ as a function of momentum $\vec{q}$ as in Fig. 3 of the main paper, but now for perfect stripes with $J / J^{\prime}=20$ and an anisotropic ad-hoc broadening in momentum space of $\gamma_{\perp}=0.18 \pi$ and $\gamma_{\|}=$ $0.09 \pi$. These results are broadly similar to that in Fig. $4 \mathrm{e}-\mathrm{h}$ of Ref. 3, where, however, the full crystal-field model for the $\mathrm{Co}^{2+}$ ions (instead of an effective spin- $1 / 2$ model) was used.

However, a closer inspection at the energy dependence of the intensities reveals strong differences. This is already seen in the comparison at selected energies as shown in Fig. 4 of the main paper, and becomes clear when considering the momentum-integrated intensity $\chi^{\prime \prime}(\omega)$, Fig. S8. While the perfect-stripe-based result has rather little intensity at low energies and a sharp saddle-point feature at $\omega_{S}$, the result for disordered stripes has large low-energy weight (note that even the anisotropy gap is almost completely smeared) and a broad saddle-point feature. As a result, the latter calculations yields only moderate intensity variations between $0.1 J S$ and $2.5 J S$, in accord with experiment (compare Fig. 4 of the main paper). In contrast, much larger intensity variations with energy arise from perfect stripes. In addition, Fig. S8 underlines the downward renormalization of $\omega_{\mathrm{S}}$ and also the overall increase of the bandwidth caused by disorder. Therefore, extracting $J S$ (or $J / J^{\prime}$ ) by matching theoretical and experimental features of the spectra (like the saddle-point location $\omega_{\mathrm{S}}$ ) requires care.

\section{SPIN-GLASS SIGNATURES}

We can characterize spin-glass behavior in our MC simulations for the spin sector by monitoring the specific heat $C$ and the magnetic correlation length $\xi_{\mathrm{S}}$ as function of temperature $T$, both shown in Fig. S9. Panel (a) shows that the specific-heat peak, which occurs near the mean-field Néel temperature in the perfect-stripe case, is strongly broadened and shifted to a lower $T_{f}$ with increasing stripe disorder. This effect is more pronounced

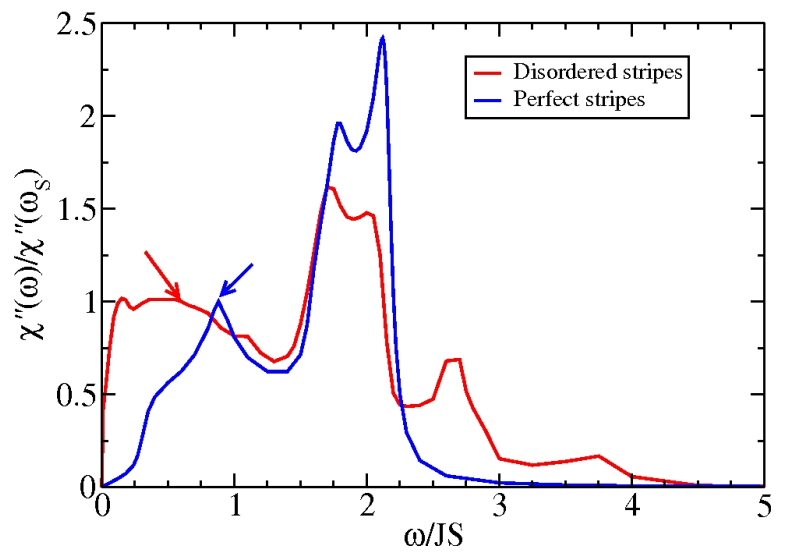

Figure S8: Momentum-integrated spectral weight as function of energy $\omega$, comparing the result from a full simulation of disordered stripes (with $\xi_{\mathrm{C}}^{\|} \simeq 5, \xi_{\mathrm{C}}^{\perp} \simeq 2$ as in Fig. $2 \mathrm{~d}$ of the main paper) to that of perfect stripes (additional momentumspace broadening does not influence this curve). In both cases $J / J^{\prime}=20$, and a Lorentzian energy broadening of $\Gamma=0.1 J S$ has been employed. The arrows denote the energy position $\omega_{\mathrm{S}}$ of the $(\pi, \pi)$ saddle point, and the intensities have been rescaled to match at $\omega_{\mathrm{S}}$. The intensity peaks in the range $1.7-2.1 J S$ originate primarily from the upper of the two spin-wave branches. ${ }^{3}$

for weakly coupled stripes, i.e., large $J / J^{\prime}$. As can be seen in panel (b), the correlation length $\xi_{\mathrm{S}}$ grows upon cooling, in particular in the temperature regime near $T_{f}$, but remains much smaller than the system size as $T \rightarrow 0$. Again, this is more pronounced for large $J / J^{\prime}$, which also causes a larger anisotropy as measured by the ratio $\xi_{\mathrm{S}}^{\|} / \xi_{\mathrm{S}}^{\perp}$.

In addition, we have also calculated the spin-glass correlation length $\xi_{\mathrm{SG}}$ from the correlations of the EdwardsAnderson order parameter. ${ }^{4,10}$ This correlation length strongly increases below $T_{f}$ as well and reaches values of the order of the system size as $T \rightarrow 0$ (not shown). Importantly, $\xi_{\mathrm{SG}}$ is always larger than $\xi_{\mathrm{S}}$ (except for very large $T$ ).

All these results are consistent with the behavior of a spin glass with short-range magnetic order and a meanfield freezing temperature $T_{f}$. Clearly, glassiness increases with increasing charge disorder and with increasing $J / J^{\prime}$, as indicated by a broader freezing regime and smaller $\xi_{\mathrm{S}}$. For large $J / J^{\prime}$, the glassy state consists of ordered AF clusters (formed by intact stripe segments of $\mathrm{Co}^{2}+$ ions) which are weakly correlated among each other (see also Fig. S10) - this justifies to call this a cluster spin glass. Note that the ground state is expected to remain glassy even in the limit of $J \sim J^{\prime}$, where the strongly disordered system corresponds to a vacancy-doped frustrated spiral magnet which displays more conventional spin-glass (instead of cluster-spin-glass) behavior.

We note that, in our two-dimensional spin model $\mathcal{H}_{\mathrm{sp}}$, a true spin-glass transition does not exist. ${ }^{11}$ Therefore, we refrain from a detailed finite-size scaling analysis of 

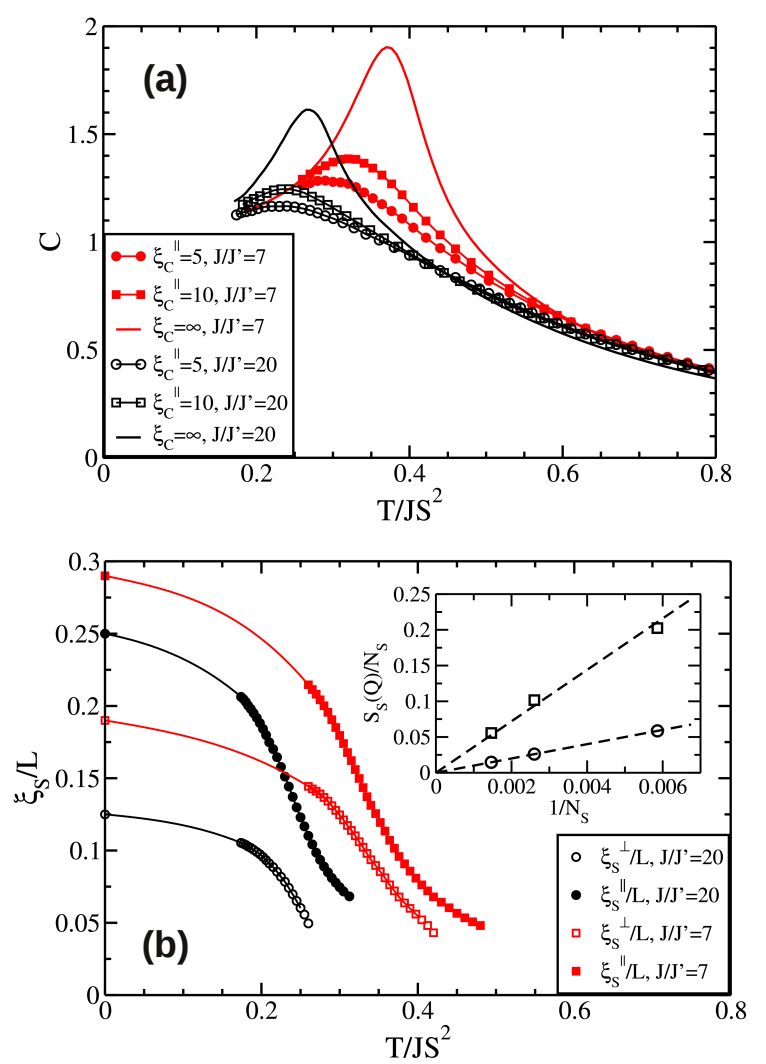

Figure S9: Characterization of glassy behavior in the spin sector, as obtained from the $\mathrm{MC}$ simulations of $\mathcal{H}_{\mathrm{sp}}$ for $L=36$. (a) Specific heat $C$ as function of temperature, for different $J / J^{\prime}$ (see legend) and different amounts of charge disorder: circles: $\xi_{\mathrm{C}}^{\|} \approx 5, \xi_{\mathrm{C}}^{\perp} \approx 2$, squares: $\xi_{\mathrm{C}}^{\|} \approx 10, \xi_{\mathrm{C}}^{\perp} \approx 5$, solid line: perfect stripes. (b) Spin correlation lengths $\xi_{\mathrm{S}}^{\|}$, $\xi_{\mathrm{S}}^{\perp}$ as function of temperature for different $J / J^{\prime}$ and $\xi_{\mathrm{C}}^{\|} \approx 5$, $\xi_{\mathrm{C}}^{\perp} \approx 2$. Here, the results at $T=0$ have been obtained from locally stable classical states. Error bars are typically smaller than the symbol size. Note that, for higher $T$ (where no data points are shown), $S_{\mathrm{S}}(\vec{q})$ does not show well-defined peaks at $\vec{Q}_{\mathrm{S}}$ but instead a broad maximum at $(\pi, \pi)$, i.e., we observe a temperature-dependent onset of incommensurability. Inset: $T=0$ spin structure factor $S_{\mathrm{S}}(\vec{q})$ at one of the ordering wavevectors $\vec{Q}_{\mathrm{S}}$ normalized by the number of spins $N_{\mathrm{S}}$ as a function of $1 / N_{\mathrm{S}}$. For both curves we have $J / J^{\prime}=20$ and different amounts of charge disorder: circles: $\xi_{\mathrm{C}}^{\|} \approx 5, \xi_{\mathrm{C}}^{\perp} \approx 2$, squares: $\xi_{\mathrm{C}}^{\|} \approx 10, \xi_{\mathrm{C}}^{\perp} \approx 5$. the thermodynamic and magnetic properties ${ }^{4,10}$ of $\mathcal{H}_{\mathrm{sp}}$ such an analysis would also be complicated by the fact that we have no straightforward way to introduce precisely the same amount of microscopic disorder at different $L$ due to the use of a non-equilibrium MC protocol. We have, however, investigated the $L$ dependence of our results for the spin structure factor $S_{\mathrm{S}}(\vec{q})$ at fixed $\xi_{\mathrm{C}}$ (which is indeed a very good estimator of disorder). For $S_{\mathrm{S}}(\vec{q})$ we essentially find no dependence on the system size in the range $L=24-48$. In particular, $S_{\mathrm{S}}\left(\vec{Q}_{\mathrm{S}}\right) / N_{\mathrm{S}} \rightarrow 0$ as $1 / N_{\mathrm{S}} \rightarrow 0$ (see the inset of Fig. $\mathrm{S} 9(\mathrm{~b}))$ indicating once more static short-range order with a vanishing magnetic order parameter. Similarly, the susceptibility $\chi^{\prime \prime}(\vec{q}, \omega)$ displays no signs of size dependence (not shown).
Finally, we illustrate the cluster-spin-glass state by showing specific saddle points of $\mathcal{H}_{\mathrm{sp}}$ in Fig. S10: these are portraits of the spin configurations of locally stable classical states. The parameters correspond to moderately and strongly disordered stripes as in Fig. 2(d,e) and Fig. S4(d,e). The configurations nicely show clusters with local AF order and essentially random relative orientations. In particular, the states are non-collinear, i.e., the small easy-axis anisotropy $\epsilon$ is less effective in the presence of disorder.
1 P. Babkevich, D. Prabhakaran, C. D. Frost, and A. T. Boothroyd, Phys. Rev. B 82, 184425 (2010).

2 L. M. Helme, A. T. Boothroyd, R. Coldea, D. Prabhakaran, C. D. Frost, D. A. Keen, L. P. Regnault, P. G. Freeman, M. Enderle, and J. Kulda, Phys. Rev. B 80, 134414 (2009).

3 A. T. Boothroyd, P. Babkevich, D. Prabhakaran, and P. G. Freeman, Nature 471, 341 (2011).

4 E. C. Andrade and M. Vojta, EPL 97, 37007 (2012).

5 J.-P. Blaizot and G. Ripka, Quantum Theory of Finite Sys- tems (MIT Press, Cambridge, MA, 1986).

6 E. R. Mucciolo, A. H. Castro Neto, and C. Chamon, Phys. Rev. B 69, 214424 (2004).

7 S. Wessel and I. Milat, Phys. Rev. B 71, 104427 (2005).

8 We employ the algorithm described in the appendix of Ref. 7.

${ }^{9}$ We note that there are no zero modes in the present discrete spin-wave calculation, because the magnetic anisotropy precludes the existence of Goldstone modes.

10 L. W. Lee and A. P. Young, Phys. Rev. B 76, 024405 
(a)

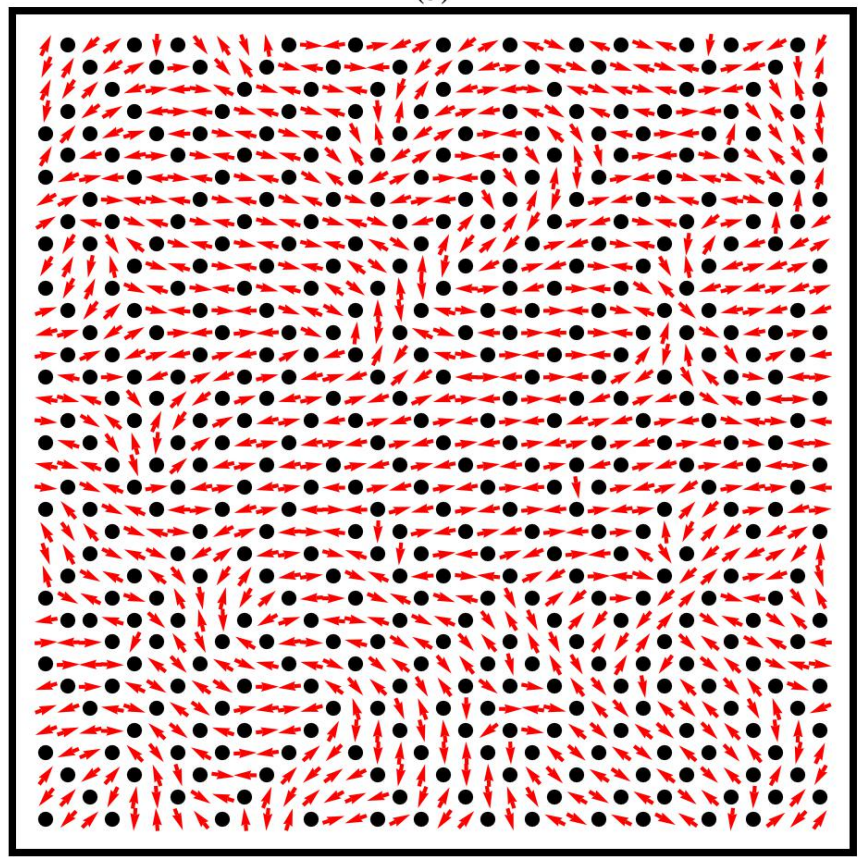

(b)

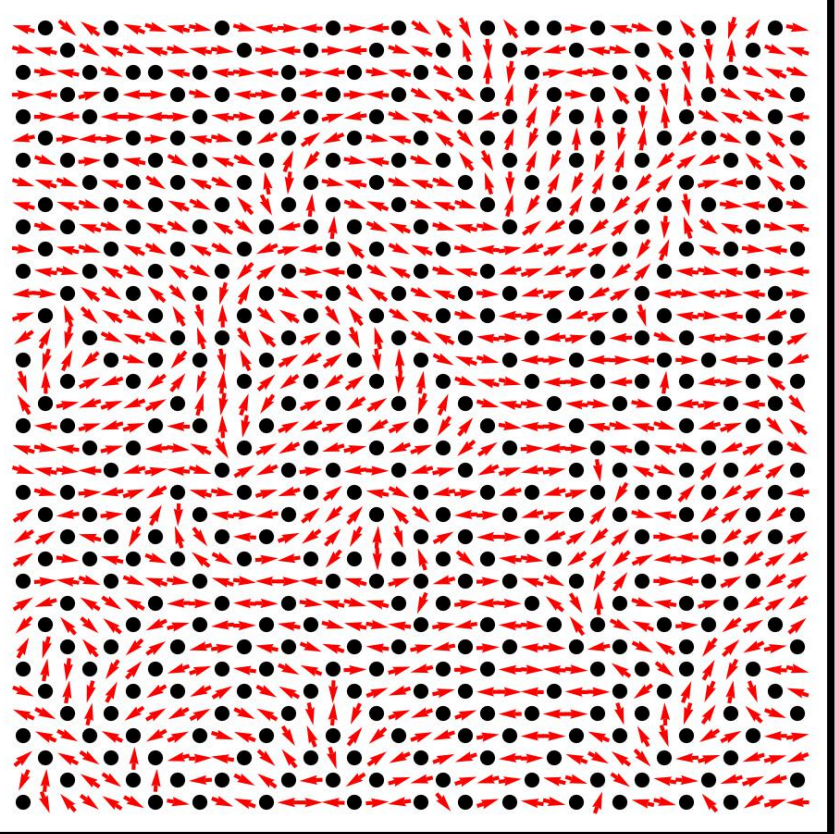

Figure S10: Real-space spin configurations in classical ground states of $\mathcal{H}_{\mathrm{sp}}$ with $L=36$, for the same parameters and charge configurations as in Fig. $\mathrm{S} 4(\mathrm{~d})\left(\xi_{\mathrm{C}}^{\|} \simeq 5, \xi_{\mathrm{C}}^{\perp} \simeq 2(\mathrm{a})\right)$ and Fig. $\mathrm{S} 4(\mathrm{e})\left(\xi_{\mathrm{C}}^{\|} \simeq 3, \xi_{\mathrm{C}}^{\perp} \lesssim 1(\mathrm{~b})\right)$. The dots show the non-magnetic sites, while the arrows show the $x$ and $y$ components of the $\vec{S}_{i}$ (the $z$ components are tiny due to the strong anisotropy caused by $\delta$ ).

(2007); D. X. Viet and H. Kawamura, ibid. 80, 064418 (2009).

11 K. H. Fischer and J. A. Hertz, Spin Glasses (Cambridge
University Press, Cambridge, 1991). 